\title{
Measurements of proton-induced radionuclide production cross sections to evaluate cosmic-ray activation of tellurium
}

\author{
A. F. Barghouty ${ }^{1}$, C. Brofferio ${ }^{2,3}$, S. Capelli ${ }^{2,3}$, M.Clemenza $^{2,3}$, O. Cremonesi ${ }^{2,3}$, \\ S.Cebrián ${ }^{4}$, E. Fiorini ${ }^{2,3, *}$, R. C. Haight ${ }^{5}$, E. B. Norman ${ }^{6,7,8}$, M. Pavan ${ }^{2,3}$, E.Previtali ${ }^{2,3}$ \\ , B. J. Quiter ${ }^{6}$, M.Sisti ${ }^{2,3}$, A. R. Smith ${ }^{7}$, and S. A. Wender ${ }^{5}$ \\ ${ }^{1}$ NASA-Marshall Space Flight Center, MSFC, AL 35812 U.S.A. \\ ${ }^{2,3}$ Dipartimento di Fisica dell' Università di Milano-Bicocca and Istituto Nazionale di Fisica Nucleare Sezione di \\ Milano-Bicocca, 20126 Milan, Italy \\ ${ }^{4}$ Universidad de Zaragoza, 50009 Zaragoza, Spain \\ ${ }^{5}$ Los Alamos National Laboratory, Los Alamos, NM 87545 U.S.A. \\ ${ }^{6}$ Nuclear Engineering Department, University of California, Berkeley, CA 94720 U.S.A. \\ ${ }^{7}$ Nuclear Science Division, Lawrence Berkeley National Laboratory, Berkeley, CA 94720 U.S.A. \\ ${ }^{8}$ Physics Division, Lawrence Livermore National Laboratory, Livermore, CA 94551 U. S. A. \\ *Corresponding author \\ E-mail address: ettore.fiorini@mib.infn.it \\ Tel.: 00390264482424 FAX: 00390264482463 \\ \#arXiv: $1010.4066 \mathrm{v} 1$
}




\section{1}

\section{INTRODUCTION}

2 Experiments designed to study rare events such as the interactions of solar neutrinos [1],

3 dark matter particles [2] or rare processes like double beta decay (DBD) [3] are carried out in

4 underground laboratories. One of the main problems in such searches is the presence in

5 various energy regions of background due to environmental radiation. The contribution due

6 to cosmic rays [4] is strongly reduced by installing the experiment underground [5],

7 sometimes with a further reduction by means of veto counters. While the local environmental

8 radiation, mainly $\gamma$-rays or neutrons, can be suitably shielded with inert materials, special

9 care should be devoted to reduce the intrinsic radioactivity of the detector itself or of the 10 material immediately surrounding it [6]. In addition to the natural primordial radioactive 11 contamination of detector material, one has to reduce the presence of radioactive nuclei 12 produced by cosmic rays before the installation underground [7]. Shipping materials by 13 airplane may be an issue due to the much higher cosmic ray flux at high altitude which could 14 be two orders of magnitude higher.

15 The cosmic-ray contribution to the intrinsic radioactivity of detectors has been predicted 16 by various authors using specific codes like COSMO [8] or general purpose codes like 17 GEANT4 [9] .To evaluate the activation cross sections for particles at cosmic ray energies, a 18 set of semi-empirical cross section formulas for proton-nucleus reactions developed by 19 Silberberg and Tsao [10,11], and are used by codes like the mentioned COSMO and the 20 recently introduced ACTIVIA [12]. To improve its reliability, the latter has been tuned to 21 measurements of activation cross sections carried out with protons at various accelerators and 22 can be applied to evaluate the effect of cosmic rays, because the fluxes [4] and cross-sections 23 [12] of cosmic-ray neutrons in altitude can be assumed to be similar to those for protons [13,

24 14]. Many of these cross-section evaluations and measurements have been carried out for 25 germanium [15-25] because of experiments with Ge diodes; others refer to different targets 
26 [26-34]. In this paper, we report extensive measurements of proton-induced radionuclide 27 production cross sections on tellurium, for which limited data exist $[12,35,36]$. The main goal 28 of this study is the identification of the radioactive isotopes that can be produced by cosmic 29 ray exposure of $\mathrm{TeO}_{2}$ cryogenic detectors (or bolometers) [37].

$30 \mathrm{TeO}_{2}$ bolometers are used to search for neutrinoless $\mathrm{DBD}$ of ${ }^{130} \mathrm{Te}$ which has a natural 31 abundance of $33.8 \%$ and a transition energy $\left(\mathrm{Q}_{\beta \beta}\right)$ of $2527.0 \mathrm{keV}[38,39]$. The best 32 sensitivity on the absolute neutrino mass, comparable to the one of ${ }^{76} \mathrm{Ge}$ and ${ }^{100} \mathrm{Mo}$ 33 experiments, has been reached with the CUORICINO experiment [40,41] based on an array 34 of 62 crystals of $\mathrm{TeO}_{2}$ with a total mass of about $40.7 \mathrm{~kg}$. To improve this result a larger scale 35 experiment is presently under construction : The Cryogenic Underground Observatory for 36 Rare Events (CUORE) $[42,43]$ will employ approximately $750 \mathrm{~kg}$ of $\mathrm{TeO}_{2}$ bolometers in a 37 search for the neutrinoless DBD of ${ }^{130}$ Te. Its aim is to reach a sensitivity on the absolute 38 neutrino mass as indicated by the results of neutrino oscillations in the inverse hierarchy 39 hypothesis. Minimizing the background in the vicinity of the $Q_{\beta \beta}$ value is crucial for the 40 success of this experiment. Thanks to the deep underground location of the apparatus (that is 41 being assembled in the underground Laboratori Nazionali del Gran Sasso, Italy) and to a 42 careful design of the gamma-ray and neutron shields [44,45], the main background sources in 43 CUORE will come from the radioactivity of the detector constructing materials $[42,43,46,47]$.

44 Among these sources, cosmogenic activated isotopes may play a relevant role. For this reason 45 a special protocol for the crystal procurement have been studied [46,47] in order to minimize 46 the exposure of $\mathrm{TeO}_{2}$ crystals to cosmic rays during growth and transportation: crystals are 47 delivered from China (where they are grown) to Italy by ship in order to avoid the larger 48 activation due by air transportation, then are immediately stored underground. The total 49 exposure to sea-level cosmic rays does not exceed 45 days (starting from growth) while the 50 storage underground is between 4 and 1 years long, depending on the production batch. 
51 To demonstrate that this procurement protocol is able to ensure acceptable low levels of 52 cosmogenic activation is not straightforward. Indeed, a direct evaluation of the activity of the 53 potentially dangerous isotopes is often impossible: whatever the technique used the sensitivity

54 is too low (as an example CUORE requirements for the $\mathrm{TeO}_{2}$ crystal contamination in ${ }^{60} \mathrm{Co}$ 55 are below one $\mu \mathrm{Bq} / \mathrm{kg}[42])$.

56 For this reason the only solution is to rely on the computation of the expected activity on the 57 basis of crystal exposure time, cosmic ray fluxes and activation cross sections.

58 2. EXPERIMENTAL SETUP

59 Exposures to the proton beams of $\mathrm{TeO}_{2}$ samples have been performed in USA at the Los 60 Alamos National Laboratory's Neutron Science Center at $0.8 \mathrm{GeV}$ and in Europe at CERN at $61 \quad 1.4$ and $23 \mathrm{GeV}$. The content of other elements in our samples was always less that a $\mu \mathrm{g} / \mathrm{g}$. 62 Before exposure, each sample was tested for the presence of relevant activities produced by 63 cosmic rays which were found to be negligible. All accelerator activation has been followed 64 by $\gamma$-ray spectroscopic analysis of the irradiated samples at different times after irradiation.

2. 1 MEASUREMENTS CARRIED OUT IN USA

66 The $0.80-\mathrm{GeV}$ irradiation was conducted in the "Blue Room" at the Los Alamos National 67 Laboratory's Neutron Science Center (LANSCE). The Te target was fabricated from 68 99.9999\% pure Te Alpha Aesar metallic powder that was pressed into a 6.9-mm thick disk 69 that had $93.4 \%$ of normal Te metal density. To confirm that the proton beam was impinging 70 entirely upon the target, a phosphor was placed in the target position before irradiation. A 71 stack of targets was placed in air and irradiated with $800 \pm 5 \mathrm{MeV}$ protons for approximately 725 minutes. The total beam fluence was monitored by a beam current integrator in order to 73 have approximately $10^{13}$ protons incident upon the target stack. The stack was made up of $746.4 \mathrm{~mm}$ thick polyethylene in front of the Te target and then an aluminium backing of $2 \mathrm{~mm}$ 75 thickness. More precise proton fluence numbers were extracted from the production of ${ }^{22} \mathrm{Na}$ 
and ${ }^{7} \mathrm{Be}$ in the monitor materials. The proton fluence deduced from the monitor foils was determined to be $(1.2 \pm 0.1) \times 10^{13} \mathrm{p} \cdot \mathrm{cm}^{-2}$. The cross sections for production of these isotopes from ${ }^{27} \mathrm{Al}$ have been previously determined using a very similar experimental setup at LANL [49], so these cross sections were used instead of evaluated data. The polyethylene monitor was analyzed using evaluated data for the $C(p, x)^{7}$ Be cross section [50]. It should be noted that the energy lost by protons while traversing the target stack was significantly less than the overall uncertainty in the beam energy and therefore the proton energies are assumed to remain constant throughout the stack.

After the bombardment at $0.8 \mathrm{GeV}$ at LANSCE, the samples were transported from Los Alamos back to Lawrence Berkeley National Laboratory (LBNL) where counting began approximately 15 days after irradiation. The samples were initially assayed with a $25 \%$ relative efficiency (with respect to that of a 7.62 x $7.62 \mathrm{~cm} \mathrm{NaI}$ detector) n-type coaxial highpurity germanium detector. The samples were counted multiple times over the course of a week in order to extract half-life information from lines that could not be readily identified.

These counting runs occurred at both $7.6 \mathrm{~cm}$ from the detector's end cap and with the sample against the end cap. Later, the targets were counted for longer periods with an $80 \%$ relative efficiency high purity germanium detector at LBNL's Low Background Facility (LBF). They were counted there intermittently for approximately one year to allow weaker long-lived lines to appear out of the Compton continuum. These counting runs occurred with samples either at $12 \mathrm{~cm}$ from the end cap or touching the end cap. All of the measurements of the targets irradiated at LANSCE were performed using ORTEC PC-based data acquisition systems. Figure 1 illustrates an energy spectrum of the Te target taken approximately 16 days after the LANSCE irradiation.

To determine the detection efficiencies for the four counting arrangements described above, calibrated point sources of ${ }^{22} \mathrm{Na},{ }^{54} \mathrm{Mn},{ }^{57} \mathrm{Co},{ }^{60} \mathrm{Co},{ }^{109} \mathrm{Cd},{ }^{137} \mathrm{Cs},{ }^{152} \mathrm{Eu}$ and ${ }^{228} \mathrm{Th}$ were 
101 used to establish a full-energy efficiency calibration curve of the detectors for point sources.

102 The thick targets that were being counted, however, were sufficiently different from point 103 sources to warrant further effort. In order to establish first-order corrections for both the 104 geometric and self-attenuating effects of the thick targets, an identical un-irradiated Te target 105 (henceforth referred to as "blank") was placed at the described distance and the point source 106 was placed behind the blank. The efficiency curves resulting from these two geometries were 107 then averaged to produce the effective efficiency curve for a given target being counted by a 108 given detector. While this method proved effective for the calibration of counting geometries 109 where the targets were further from the detector, coincident summing of lines prevented it 110 from being practical when the targets were placed directly against the end caps of the 111 detectors. In this case we employed a method similar to that described by Gmuca and 112 Ribansky [51]. An efficiency curve for a geometry with the source further from the detector 113 is determined as above, then using only the sources with non-coincident lines, ${ }^{57} \mathrm{Co},{ }^{109} \mathrm{Cd}$, $114{ }^{137} \mathrm{Cs}$, and ${ }^{54} \mathrm{Mn}$, the ratio of efficiencies at the two geometries is determined and a curve is fit 115 to that ratio. Then the longer-distance efficiency curve is multiplied by the aforementioned 116 curve resulting in the final efficiency curve for these close geometries.

117 A brief inspection of the spectra shown in Fig. 1 allows one to gain an idea as to how many 118 lines were present in the spectra. Each line was identified by energy and half-life. Where 119 lines could be attributed to multiple isotopes, other lines were searched for to determine if one 120 121 confirm the presence of an isotope because it only had a single line or its other lines were also 122 irresolvable, information from the half-life was employed. Cross sections were determined 123 using the extracted $\gamma$-ray peak areas, measured $\gamma$-ray detector efficiencies, tabulated $\gamma$-ray 124 intensities [51], measured target thickness, measured proton fluence and the start and stop 125 times of the $\gamma$-ray counting. 


\section{2 MEASUREMENTS CARRIED OUT IN EUROPE}

Crystals of $\mathrm{TeO}_{2}$ with natural isotopic composition of tellurium [48] were exposed in air to two proton beams of different energies at CERN. Details on the samples and on the exposures are reported in Table I. A single exposure of a telluride crystal of $0.457 \mathrm{~g}$ mass and irregular shape was performed at the $1.4 \mathrm{GeV}$ beam of ISOLDE using the RABBIT system [52]. The cross section of the beam was approximately circular with a radius at half maximum of $\sim 2$ $\mathrm{cm}$, thus totally covering the sample. The geometrical beam profile was taken into account to evaluate the effective proton fluence on the target. Due to the small thickness of the target, energy loss in it was negligible. The total beam fluence was monitored by a beam current integrator and known to within 10\%.

Two exposures were also carried out in air with the $23 \mathrm{GeV}$ beam at the IRRADIATION 1 Facility at the CERN PS East Hall $[53,54]$. The beam fluence was monitored with a current integrator and tested by activation of a $100 \mu \mathrm{m}$ thick Al foil. The overall uncertainty on the fluence was $10 \%$. The first exposure was carried out with a crystal of $\mathrm{TeO}_{2}$ of irregular shape with a mass of $0.428 \mathrm{~g}$ and a low proton fluence in order to pre-evaluate the expected activity after irradiation. Also in this case the cross section of the beam did totally covered the sample and the beam profile was used to evaluate the effective proton fluence on the target. The loss of protons in the crystal is negligible.

A second exposure was performed later with a larger $\mathrm{TeO}_{2}$ crystal of 23 x $22 \mathrm{~mm}$ sides and $1.6 \mathrm{~mm}$ thickness. As for the exposure at $1.4 \mathrm{GeV}$, the energy loss of the protons inside the target was negligible. The cross section of the beam was approximately circular with a radius at half maximum radius of $\sim 8 \mathrm{~mm}$. Since the beam did not cover the entire target the effective fluence through it was calculated taking into account, also in this case, the beam profile. 
For radiation safety reasons and in order to avoid transportation from the irradiation zones, $\gamma$-ray spectroscopy for the CERN irradiated samples was first performed for period of a few months after exposure by the Irradiation Facility of CERN with standard CANBERRA Ge spectrometers with relative efficiency of up to $40 \%$ (with respect to that of a $7.62 \times 7.62 \mathrm{~cm}$ $\mathrm{NaI}$ detector). However, since the presence of long-living isotopes is more relevant for a lowbackground underground experiments like CUORE, the sample irradiated at $1.4 \mathrm{GeV}$ and the second one of those irradiated at $23 \mathrm{GeV}$ were shipped to Milan years after the exposure, when the activity due to the short-living isotopes had sufficiently decreased.

The irradiated samples were then analyzed with two high purity germanium detectors, one of $\sim 30 \%$ and the other one of $\sim 60 \%$ relative efficiency. The energy resolutions of the two detectors at $1.33 \mathrm{MeV}$ were $1.77 \mathrm{keV}$ and $2.01 \mathrm{keV}$ FWHM, respectively. All detectors were operated in the low-radioactivity laboratory of the Italian National Institute for Nuclear Physics (INFN) and of the University of Milano-Bicocca in the basement of the Territorial and Environmental Science Department.

Monte Carlo simulations were done in order to correctly evaluate the HPGe absolute detection efficiency $\left(\varepsilon_{\mathrm{abs}}\right)$ considering the relative position of the samples with respect to the detector. The efficiency of the detector for the analyzed radioisotopes was reconstructed with the aim of evaluate all possible aspects, especially those connected with coincidence summing effect that can influence the full energy peak efficiency for some radionuclides (for example: the complete decay scheme of ${ }^{60} \mathrm{Co},{ }^{65} \mathrm{Zn},{ }^{54} \mathrm{Mn},{ }^{102 \mathrm{~m}} \mathrm{Rh}$ and ${ }^{110 \mathrm{~m}} \mathrm{Ag}$ were considered).

The software utilized named Jazzy, is based on GEANT4 [9]. This code has been tested by exposing our detectors to several certified gamma ray sources (point like and extended) of the Italian Authority ENEA and found to reproduce the entire spectrum (50 keV up to $2.5 \mathrm{MeV}$ ) within $5 \%$ error [6]. Taking into account the irregular shape of samples, it was assumed a systematic error of $10 \%$ in the overall gamma ray efficiency. 

the exposures at 1.4 and $23 \mathrm{GeV}$ are reported in Figures 2 and 3, respectively. They clearly show the presence of long-living isotopes produced by proton activation. Identifications of the major peaks produced by the irradiation are also indicated (Figures $2 \mathrm{~b}$ and $3 \mathrm{~b}$ ).

\section{RESULTS AND DISCUSSION}

Our primary aim was to determine the production cross sections of long-lived isotopes, such as ${ }^{60} \mathrm{Co}$, and other long-living nuclei, whose presence could contribute to the background in the energy region of interest $\left(2527 \mathrm{keV}\right.$ in the case of ${ }^{130} \mathrm{Te}$ neutrinoless DBD). The quoted errors (90\% c.1.) include in addition to statistics also the uncertainties on proton fluence and detector efficiency. The gamma rays produced by the decay of ${ }^{60} \mathrm{Co}$ in the proton-irradiated Te targets are initially obscured by the activity of short-lived isotopes and can only be reliably measured months or years after the exposures. For completeness, however, we also report our results for relatively short-lived isotopes, which could play a role in the first period of the underground experiment, especially if the material was shipped by airplane.

The experimental results obtained from the irradiations performed at LANSCE and CERN are reported in Tables II and III, for long and short-living isotopes, respectively. We have calculated and also reported in the Tables the production cross sections calculated using the latest version of the semi-empirical formulae of Silberberg and Tsao (S\&T) [10,11] (using the YIELDX routine). We note that the code only calculates cross sections for a specific daughter to be produced from a specific target. Thus, to determine each production cross section as defined above, calculations must be performed for each naturally occurring target isotope yielding the daughter nuclide as well as all short-lived precursors that decay to the daughter of interest. In the S\&T calculations, no discrimination is made between meta-stable and ground states. 
Before comparing our results with predictions, we would like to stress that the evaluation 200 of Silberberg and Tsao is based on the hypothesis of activation by spallation [55]. This is

\section{CONCLUSIONS}

The above reported cross sections can be used to evaluate the contribution by cosmic ray activation of $\mathrm{TeO}_{2}$ crystals to the background of CUORE. Limiting ourselves to long living nuclei (Table II), the only two isotopes able to contribute to the background in the Region Of 
224 Interest (ROI) for the ${ }^{130}$ Te Double Beta Decay study are ${ }^{60} \mathrm{Co}$ and ${ }^{110 \mathrm{~m}} \mathrm{Ag}$. Indeed all the

225

others have a maximum energy release in the crystal far below the ROI. A rough evaluation of the initial activity of these isotopes in $\mathrm{TeO}_{2}$ crystals is obtained using the cross sections reported in Table II and binning the cosmic ray spectrum at sea level accordingly. For an average exposure of 45 days, we obtain an activity of about $2 \times 10^{-8} \mathrm{~Bq} / \mathrm{kg}$ in ${ }^{60} \mathrm{Co}$ and $4 \times 10^{-}$ ${ }^{6} \mathrm{~Bq} / \mathrm{kg}$ in ${ }^{110 \mathrm{~m}} \mathrm{Ag}$. These activities will decrease before the start of the experiment (the storage time varies between 5 and 1 years depending on the crystal production batch) by a factor 2 (for ${ }^{60} \mathrm{Co}$ ) and 9 (for ${ }^{110 \mathrm{~m}} \mathrm{Ag}$ ) leading to a global contribution in the ROI that - according to preliminary evaluations - is at least one order of magnitude below the target counting rate for CUORE.

\section{ACKNOWLEDGMENTS}

We would like to express our gratitude to the ISOLDE Collaboration and in particular to Alexander Helert and Ulli Koester and to the ISOLDE Technical group for assistance and support. We are also grateful to the CERN IRRADIATION FACILITY and in particular to Maurice Glaser for help and advice in the exposures at $23 \mathrm{GeV}$. This work was supported in part by the U. S. Department of Energy under contract numbers DE-AC52-07NA27344 at LLNL and DE-AC02-05CH11231 at LBNL.

\section{REFERENCES}

[1] W. C. Haxton, arXiv:0808.0735 and Encyclopedia of Applied High Energy and Particle Physics (ed. R Stock) (2008) 221.

[2] L. Baudis, arXiv:0711.3788v1 and Proc. SUSY07 (Karlsruhe, 2007) 198.

[3] F. T. Avignone III, S. R. Elliott, J. Engel, Rev. Mod. Phys. 80 (2008) 481.

[4] C. Amsler, et al., Review of Particle Physics, Phys. Lett. B 667 (2008) 1.

[5] S. Niese, Radioactivity in the Environment 11 (2008) 209.

[6] C. Bucci et al. , Eur. Phys. Journal A 41(2009) 155. 
[7] G. Heusser, Annual. Rev. Nucl. Part. Sci. 45 (1995) 543.

[8] C. J. Martoff and P.D. Lewin, Comp. Phys. Commun. 72 (1992) 96.

[9] S. Agostinelli et al, Nucl. Instr. and Meth. A 506 (2003) 250.

[10] R. Silberberg, C.H. Tsao, Astrophys. J. Suppl. 220 (I) (1973) 315; Astrophys. J. Suppl. 220 (II) (1973) 335; Physics Reports 191 (1990) 351.

[11] R. Silberberg, C.H. Tsao and A.F. Barghouty. Astrophys. J. 501 (1998) 911.

[12] J. J. Back and Y. A. Ramachers: Nucl. Instrum. Meth. A 586 (2008) 286-294.

[13] X. Hou, Radioactivity in the Environment, 11 (2008) 371.

[14] G. Heusser, M. Laubenstein, H. Neder, Radioactivity in the Environment, 8 (2006) 495.

[15] I. Barabanov et al., Cosmogenic activation of Germanium and its reduction for low background experiments, nucl-ex/0511049 (2005) and Radioactivity (2007) 1. Nucl. Instrum. Meth. B 251 (2006) 115-120.

[16] H. Gómez, S. Cebrián, J. Morales, J.A. Villar, Astropart. Phys. 28 (2007) 435.

[17] S. Fiorucci et al, Astropart. Phys. 28 (2007) 143.

[18] F. T. Avignone et al., Nucl. Phys. B Proc. Suppl. 28 (1) (1992) 280.

[19] M. Blann, Phys. Rev. C 54 (1996) 1341; M. Blann, M.B. Chadwick, Phys. Rev. C 57 (1998) 233.

[20 ] H. V. Klapdor-Kleingrothaus, et al., Nucl. Instr. and Meth. A 481 (2002) 149.

[21] H. S. Miley, et al., Nucl. Phys. B Proc. Suppl. 28 (I) (1992) 212.

[22] D. M. Mei, Z. . Yin, S. R. Elliott Astropart. Phys. 31 (2009) 417-420.

[23] Mei, D.M.; Elliott, S. R.; Hime, A., et al., Phys. Rev. C 77 (2008) 054614.

[24] S. R. Elliott et al., Phys. Rev. C 82 (2010) 054610.

[25] A study on activation in germanium by neutrons due to muon interactions underground is reported in D. M. Mei and A. Hime: Muon-induced background study for underground laboratories, astro-ph/0512125 6 Dec. 2005 Phys. Rev. D 73 (2006) 053004. 
[26] J. A. Formaggio, C. J. Martoff, Ann. Rev. Nucl. Part. Sci. 54 (2004) 361.

275

4

[27] E. Porras et al, Nucl. Instr. and Meth. B 160 (2000) 73.

[28] Yu. E. Titarenko et al., Phys.Rev. C 78 (2008) 034615.

[29] Yu. E. Titarenko et al.: Verification of high-energy transport code $\mathrm{s}$ on the basis of activation data arXiv:1106.0054.

[30] S. Takaca et al., Nucl. Instr. and Meth. B 188 (2002) 106.

[31] S. Takaca et al., Nucl. Instr. and Meth. B 251 (2006) 56.

[32] L. Baudis and R.W. Schnee, Dark Matter experiments, NUSL White Paper, 2002.

[33] A. C. Rester et al., Nucl. Instr. and Meth. A 297 (1990) 258.

[34] V. E. Giuseppe, M. Devlin, S. R. Elliott, et al., Physical Review C 79 (2009) 054604.

[35] D.W. Bardayan et al., Phys. Rev. C 55 (1997) 820.

[36] E.B. Norman et al, Nucl. Phys. B (Proc.Suppl.) 143 (2005) 508.

[37] N. Booth, B. Cabrera and E. Fiorini, Ann. Rev. Nucl. Part. Sci. 46(1996) 471; C. Enns and D. McCammon, J. Low Temp.Phys 151 (2008) 5.

[38] N. D. Scielzo et al., Phys. Rev. C 80 (2009) 025501.

[39] M. Redshaw et al., Phys. Rev. Lett. 102 (2009) 212502.

[40] E. Andreotti et al, Astropart. Phys. 34 (2011) 822

[41] C. Arnaboldi et al., Phys. Rev. C78 (2008) 035502

[42] C. Arnaboldi et al., Nucl. Instrum. \& Meth. A 518 (2004) 775.

[43] M. Pedretti et al., International Journal of Modern Physics A. 23 (20089) 3395.

[44] F. Bellini et al., Astroparticle Physiscs 33 (2010) 169.

[45] E. Andreotti et al., Astroparticle Physiscs 34 (2010) 18.

[46] F. Alessandria et al., Astroparticle Physics 35 (2012) 839.

[47] M. Pavan et al., Eur. Phys. Journal A 36 (2008) 159

[48] C. Arnaboldi et al. Journal of Crystal Growth 312 (2010) 2999. 
[49] T. N. Taddeucci et al., Phys. Rev. C 55 (1997) 1551.

300 [50] J. B. Cumming, Ann. Rev. of Nuc. and Part. Sci. Vol. 13 (1963) 261.

301 [51] S. Gmuca, S. and I. Ribansky, Nucl. Instr. and Meth. 202 (1982) 435.

302 [52] E. Kugler, Hyperfine Interactions 129 (2000) 23.

303 [53] M. Moll and M. Glaser: Proton and neutron irradiation facilities in the CERN East Hall304 EP common project, presented to the EP Technical Coordinator meeting on 23.9.2002.

305 [54] M. Glaser et al., Nucl. Instr. and Meth. A 426 (1999).

306 [55] M. N. Andronenko, L. N. Andronenko and W. Neubert, Eur. Phys.J. A 31 (2007). 


\begin{tabular}{|c|c|c|c|c|c|}
\hline $\begin{array}{c}\text { Proton } \\
\text { Energy } \\
(\mathrm{GeV})\end{array}$ & $\begin{array}{l}\text { Fluence } \\
\left(\mathbf{p} / \mathrm{cm}^{2}\right)\end{array}$ & $\begin{array}{c}\text { Mass } \\
\mathrm{TeO}_{2} \\
(\mathrm{~g})\end{array}$ & $\begin{array}{c}\text { Mass } \\
\text { Te } \\
(g)\end{array}$ & $\begin{array}{c}\text { Delay } \\
\text { Time } \\
\text { (h) }\end{array}$ & $\begin{array}{l}\text { Measurement } \\
\text { Live Time } \\
\text { (s) }\end{array}$ \\
\hline 1.4 (a) & $1.5 \times 10^{15}$ & 0.457 & 0.365 & 1240.5 & 297022 \\
\hline 1.4 (b) & $1.5 \times 10^{15}$ & 0.457 & 0.365 & 4827.5 & 251358 \\
\hline $1.4(\mathrm{c})$ & $1.5 \times 10^{15}$ & 0.457 & 0.365 & 40124.5 & 583178 \\
\hline $23(0)$ & $9.41 \times 10^{10}$ & 0.424 & 0.339 & 848.2 & 50400 \\
\hline 23 (a) & $6.1 \times 10^{12}$ & 51.11 & 40.9 & 2224 & 1097 \\
\hline $23(\mathrm{~b})$ & $6.1 \times 10^{12}$ & 51.11 & 40.9 & 2591 & 50400 \\
\hline 23 (c) & $6.1 \times 10^{12}$ & 51.11 & 40.9 & 13606 & 200000 \\
\hline $23(d)$ & $6.1 \times 10^{12}$ & 51.11 & 40.9 & 24793 & 861228 \\
\hline
\end{tabular}

TABLE 1. CERN Irradiations data and delay and live times of Gamma ray spectrometry measurements

\begin{tabular}{|c|c|c|c|c|c|c|c|c|c|}
\hline Isotope & $\begin{array}{l}\text { Half } \\
\text { Life } \\
\text { (d) }\end{array}$ & $\begin{array}{c}\text { Gamma } \\
\text { Ray } \\
\text { Energy } \\
\text { (keV) }\end{array}$ & $\begin{array}{l}\text { Decay } \\
\text { Mode }\end{array}$ & $\begin{array}{c}\sigma \text { Expt. } \\
0.8 \mathrm{GeV} \\
(\mathrm{mb})\end{array}$ & $\begin{array}{c}\sigma S \& T \\
0.8 \\
\text { GeV } \\
(\mathrm{mb})\end{array}$ & $\begin{array}{c}\sigma \text { Expt. } \\
1.4 \mathrm{GeV} \\
(\mathrm{mb})\end{array}$ & $\begin{array}{c}\sigma \mathrm{S} \& \mathrm{~T} \\
1.4 \mathrm{GeV} \\
(\mathrm{mb})\end{array}$ & $\begin{array}{c}\sigma \text { Expt. } \\
23 \mathrm{GeV} \\
(\mathrm{mb})\end{array}$ & $\begin{array}{c}\sigma \mathrm{S \& T} \\
23 \mathrm{GeV} \\
(\mathrm{mb})\end{array}$ \\
\hline${ }^{54} \mathrm{Mn}$ & 312 & 1377 & $\mathrm{EC}$ & $0.04 \pm 0.02$ & 0.11 & & 0.40 & $1.5 \pm 0.3$ & 4.0 \\
\hline${ }^{57} \mathrm{Co}$ & 272 & 836 & $\mathrm{EC}$ & $0.05 \pm 0.01$ & 0.20 & $0.15 \pm 0.05$ & 0.73 & $0.87 \pm 0.09$ & 1.1 \\
\hline${ }^{60} \mathrm{Co}$ & 1923.6 & 2824 & $\beta^{-}$ & $0.09 \pm 0.04$ & 0.22 & $0.20 \pm 0.04$ & 0.77 & $0.75 \pm 0.08$ & 1.15 \\
\hline${ }^{65} \mathrm{Zn}$ & 244 & 1352 & $\mathrm{EC}, \beta^{+}$ & $0.11 \pm 0.02$ & 0.46 & $1.2 \pm 0.3$ & 1.5 & $1.8 \pm 0.2$ & 2.4 \\
\hline${ }^{75} \mathrm{Se}$ & 120 & 864 & $\mathrm{EC}$ & $0.26 \pm 0.08$ & 1.1 & $3.2 \pm 0.3$ & 2.7 & $3.1 \pm 0.3$ & 4.3 \\
\hline${ }^{88} \mathrm{Y}$ & 107 & 3623 & $\mathrm{EC}, \beta^{+}$ & $3.1 \pm 1.2$ & 1.3 & $4.6 \pm 0.8$ & 5.0 & $4.2 \pm 0.6$ & 3.1 \\
\hline${ }^{102} \mathrm{Rh}$ & 207 & $\begin{array}{l}2323 \\
1151 \\
\end{array}$ & $\begin{array}{c}\mathrm{EC}, \beta^{+} \\
\beta^{-}\end{array}$ & $4.9 \pm 1.2$ & 7.9 & $1.5 \pm 0.3$ & 11 & & 5.8 \\
\hline${ }^{102 \mathrm{~m}} \mathrm{Rh}$ & 1058.5 & 2323 & $\mathrm{EC}, \beta^{+}$ & $4.0 \pm 0.6$ & 1.3 & $2.4 \pm 0.5$ & & $1.5 \pm 0.2$ & \\
\hline${ }^{110 \mathrm{~m}} \mathrm{Ag}$ & 250 & 2892 & $\beta^{-}$ & $24.6 \pm 3.7$ & 16.0 & $1.9 \pm 0.3$ & 1.2 & $0.88 \pm 0.59$ & 0.64 \\
\hline${ }^{113} \mathrm{Sn}$ & 115 & 1036 & $\mathrm{EC}$ & $12.4 \pm 1.9$ & 19.5 & $27 \pm 5$ & 19 & & 11 \\
\hline
\end{tabular}

TABLE 2. Results for activation of isotopes with half life $>100$ days 


\begin{tabular}{|c|c|c|c|c|c|c|c|c|c|}
\hline Isotope & $\begin{array}{l}\text { Half } \\
\text { Life } \\
\text { (d) }\end{array}$ & $\begin{array}{c}\text { Gamma } \\
\text { Ray } \\
\text { Energy } \\
\text { (keV) }\end{array}$ & $\begin{array}{l}\text { Decay } \\
\text { Mode }\end{array}$ & $\begin{array}{c}\sigma \text { Expt. } \\
0.8 \mathrm{GeV} \\
(\mathrm{mb})\end{array}$ & $\begin{array}{c}\sigma \mathrm{S} \& \mathrm{~T} \\
0.8 \mathrm{GeV} \\
(\mathrm{mb})\end{array}$ & $\begin{array}{c}\sigma \text { Expt. } \\
1.4 \mathrm{GeV} \\
(\mathrm{mb})\end{array}$ & $\begin{array}{c}\sigma \mathrm{S} \& \mathrm{~T} \\
1.4 \mathrm{GeV} \\
(\mathrm{mb})\end{array}$ & $\begin{array}{c}\sigma \text { Expt. } \\
23 \mathrm{GeV} \\
(\mathrm{mb})\end{array}$ & $\begin{array}{c}\sigma \mathrm{S \& T} \\
23 \mathrm{GeV} \\
(\mathrm{mb})\end{array}$ \\
\hline${ }^{46} \mathrm{Sc}$ & 83.8 & 2367 & $\beta^{-}$ & $0.05 \pm 0.01$ & 0.11 & & 0.29 & $1.5 \pm 0.3$ & 2.9 \\
\hline${ }^{48} \mathrm{~V}$ & 16 & 4012 & $\mathrm{EC}, \beta^{+}$ & & & & 0.12 & $24 \pm 5$ & 1.2 \\
\hline${ }^{56} \mathrm{Co}$ & 77.3 & 4566 & $\mathrm{EC}, \beta^{+}$ & $0.10 \pm 0.02$ & 0.18 & & 0.04 & $0.18 \pm 0.03$ & 0.40 \\
\hline${ }^{58} \mathrm{Co}$ & 70.9 & 2307 & $\mathrm{EC}, \beta^{+}$ & $0.04 \pm 0.01$ & 0.38 & $0.5 \pm 0.1$ & 1.45 & $1.7 \pm 0.3$ & 2.2 \\
\hline${ }^{59} \mathrm{Fe}$ & 44.5 & 1565 & $\beta^{-}$ & & & $0.3 \pm 0.1$ & 0.20 & $0.74 \pm 0.17$ & 0.47 \\
\hline${ }^{83} \mathrm{Rb}$ & 86.2 & 909 & $\mathrm{EC}$ & $1.6 \pm 0.2$ & 2.6 & & 4.1 & $5.3 \pm 0.8$ & 3.8 \\
\hline${ }^{84} \mathrm{Rb}$ & 32.8 & 2681 & $\mathrm{EC}, \beta^{+}$ & $0.40 \pm 0.06$ & 0.4 & $4.8 \pm 0.8$ & 1.7 & $4.2 \pm 0.7$ & 1.6 \\
\hline${ }^{85} \mathrm{Sr}$ & 64.8 & 1065 & $\mathrm{EC}$ & $2.1 \pm 0.3$ & 3.1 & & 6.1 & $5.4 \pm 0.9$ & 4.8 \\
\hline${ }^{88} \mathrm{Zr}$ & 83.4 & 673 & $\mathrm{EC}$ & & & & 7.0 & $2.8 \pm 0.6$ & 4.2 \\
\hline${ }^{87} \mathrm{Y}$ & 3.3 & 1862 & $\mathrm{EC}, \beta^{+}$ & $2.9 \pm 0.4$ & 3.2 & & 2 & & \\
\hline${ }^{95} \mathrm{Nb}$ & 34.97 & 926 & $\beta^{-}$ & & & & 1.2 & $1.5 \pm 0.5$ & 0.58 \\
\hline${ }^{89} \mathrm{Zr}$ & 3.3 & 2833 & $\mathrm{EC}, \beta^{+}$ & $3.6 \pm 0.5$ & 4.7 & & & & \\
\hline${ }^{92 \mathrm{~m}} \mathrm{Nb}$ & 10.2 & 2006 & $\mathrm{EC}, \beta^{+}$ & $0.23 \pm 0.03$ & 3.6 & & & & \\
\hline${ }^{95} \mathrm{Nb}$ & 34.97 & 926 & $\beta^{-}$ & $0.85 \pm 0.17$ & 0.5 & & & & \\
\hline${ }^{95 \mathrm{~m}} \mathrm{Tc}$ & 61 & 1691 & $\mathrm{EC}, \beta^{+}$ & $0.46 \pm 0.07$ & 7.6 & & & & \\
\hline${ }^{100} \mathrm{Pd}$ & 3.6 & 361 & $\mathrm{EC}$ & $6.4 \pm 1.1$ & 2.7 & 2 & & & \\
\hline${ }^{103} \mathrm{Ru}$ & 39.3 & 763 & $\beta^{-}$ & $1.4 \pm 0.2$ & 0.6 & & 0.82 & $19 \pm 3$ & 0.37 \\
\hline${ }^{105} \mathrm{Ag}$ & 41.3 & 1345 & $\mathrm{EC}$ & $10.1 \pm 1.7$ & 19.7 & & 15 & $3.7 \pm 1$ & 9.3 \\
\hline${ }^{111} \mathrm{In}$ & 2.8 & 865 & $\mathrm{EC}$ & $16.4 \pm 3.0$ & 30.4 & & & & \\
\hline${ }^{114 \mathrm{~m}} \mathrm{In}$ & 49.5 & 1452 & $\mathrm{EC}, \beta^{+}$ & $11.6 \pm 2.3$ & 3.7 & $9.2 \pm 0.9$ & 3.1 & $8.0 \pm 1,2$ & 1.9 \\
\hline
\end{tabular}

TABLE 3. Results for activation of isotopes with half life $<100$ days 


\section{FIGURES}

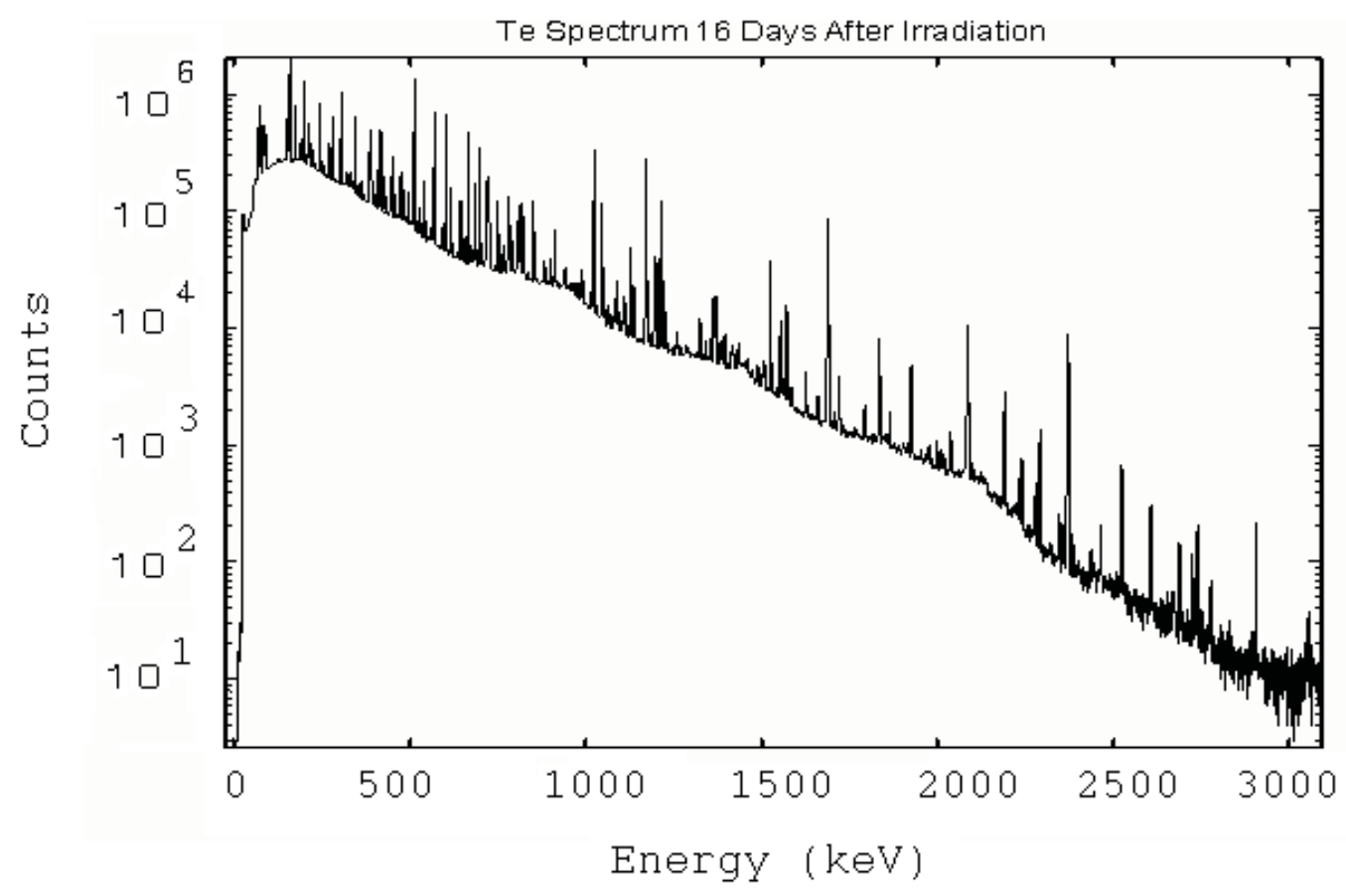

FIGURE 1: $\gamma$-ray spectrum recorded 16 days after the LANSCE irradiation with $0.80 \mathrm{GeV}$ protons. 


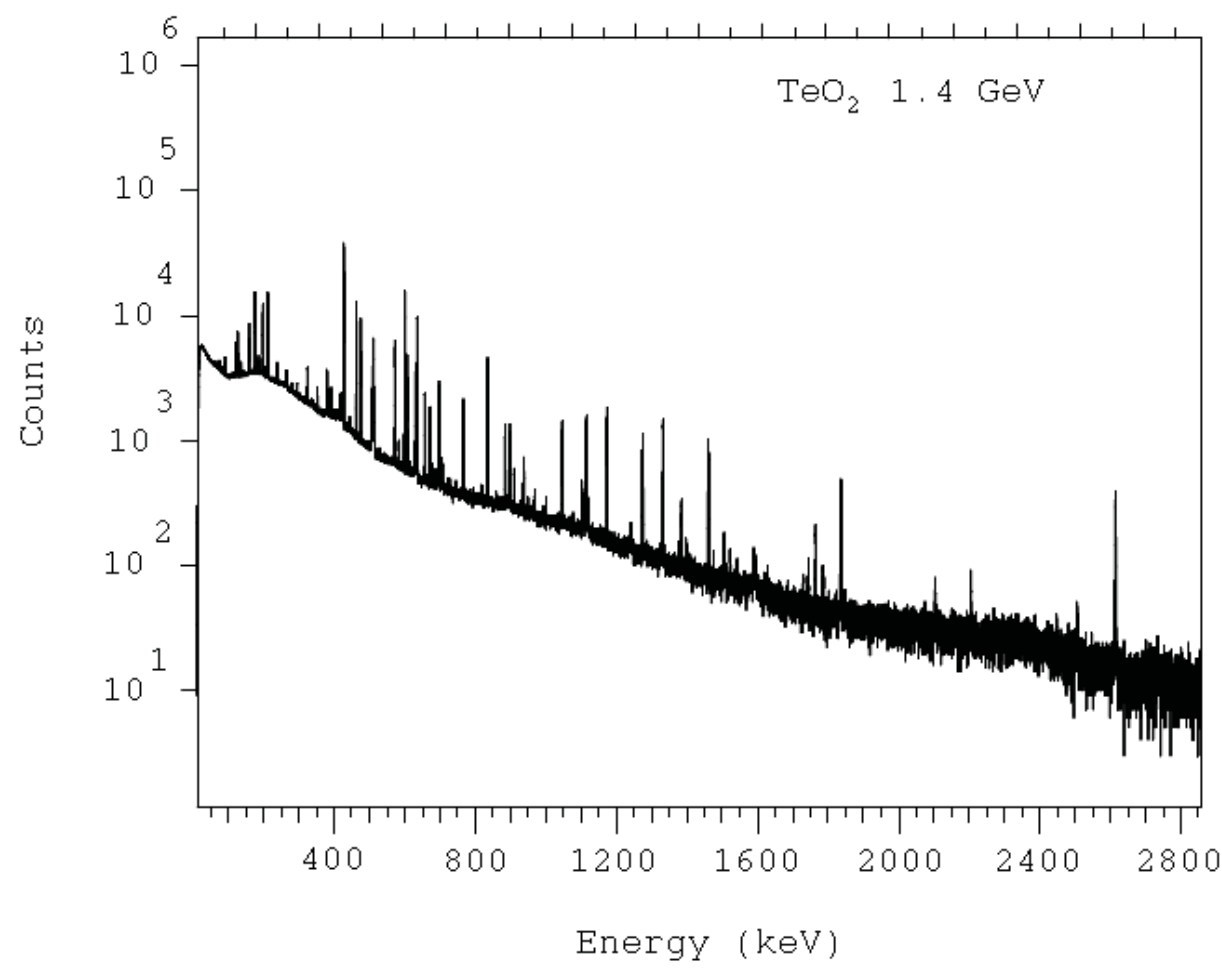

FIGURE 2(a): $\gamma$-ray spectrum (583178 sec live time) recorded for the sample irradiated at 1.4 . GeV 4.58 years after irradiation: complete spectrum 


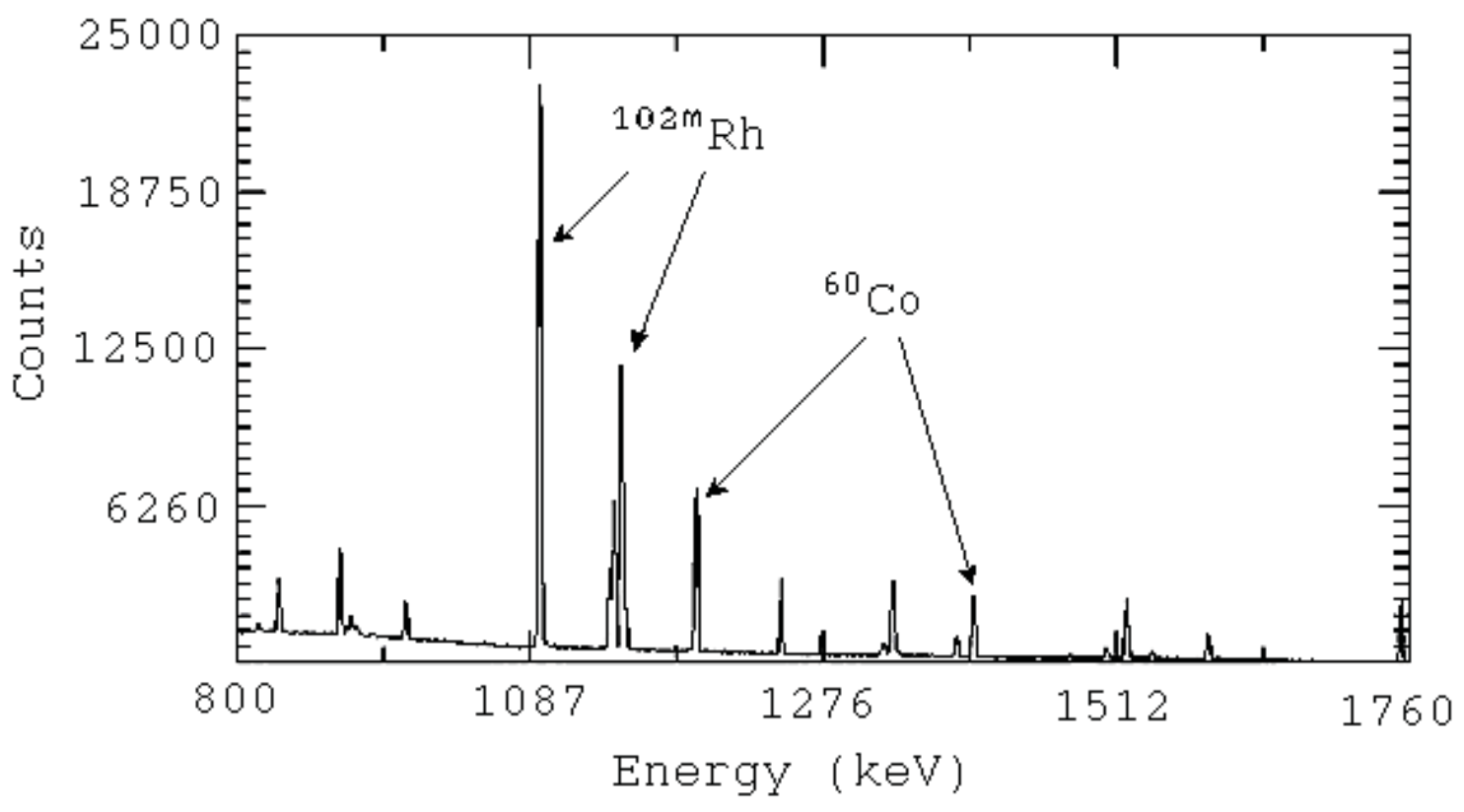

FIGURE 2(b): $\gamma$-ray spectrum (583178 sec live time) recorded for the sample irradiated at 1.4 . GeV 4.58 years after irradiation: peaks of interest. 


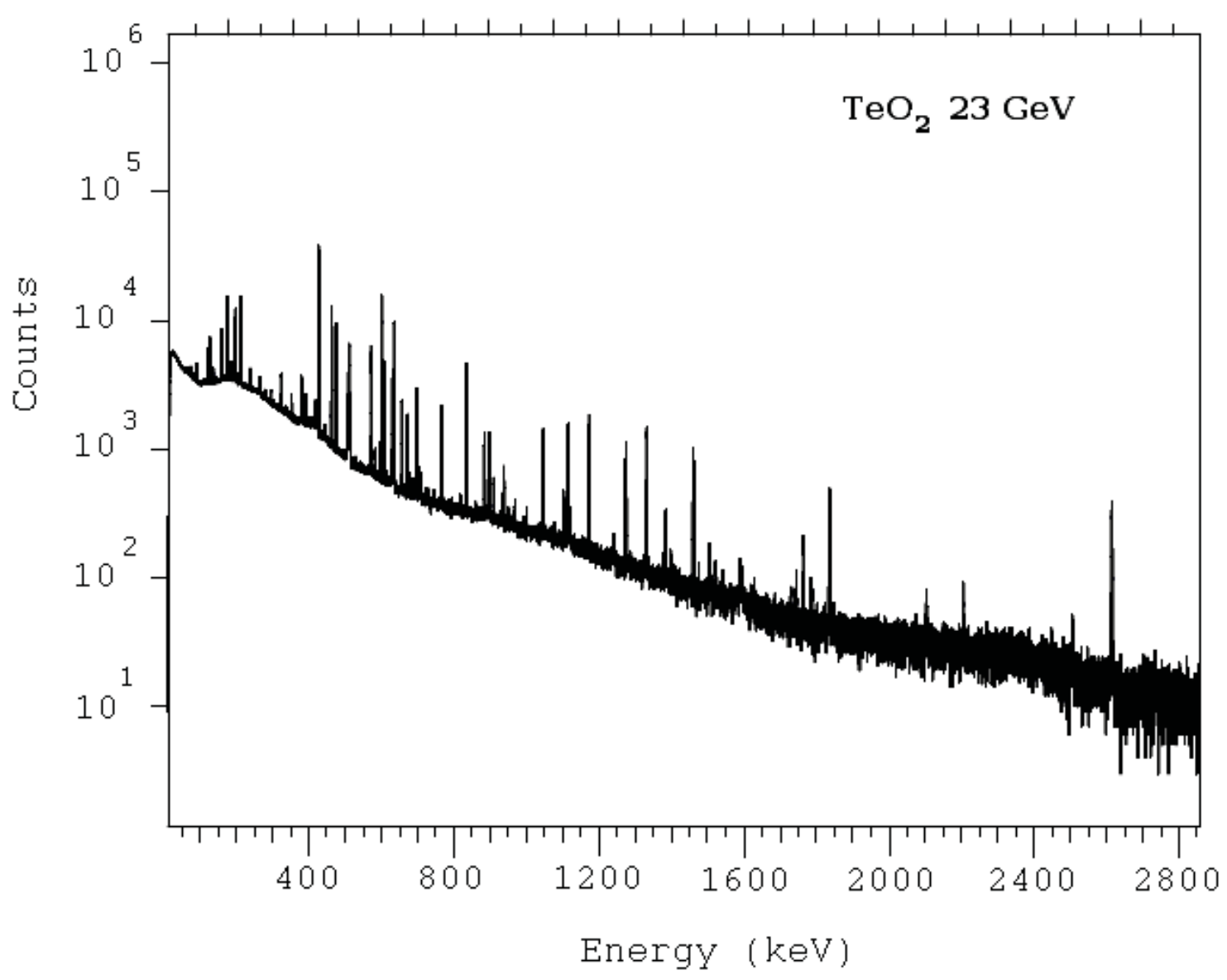

FIGURE 3(a): $\gamma$-ray spectrum (861228 sec live time) recorded for the sample irradiated at $23 \mathrm{GeV} 2.83$ years after irradiation: complete spectrum; 


\section{ACCEPTED MANUSCRIPT}

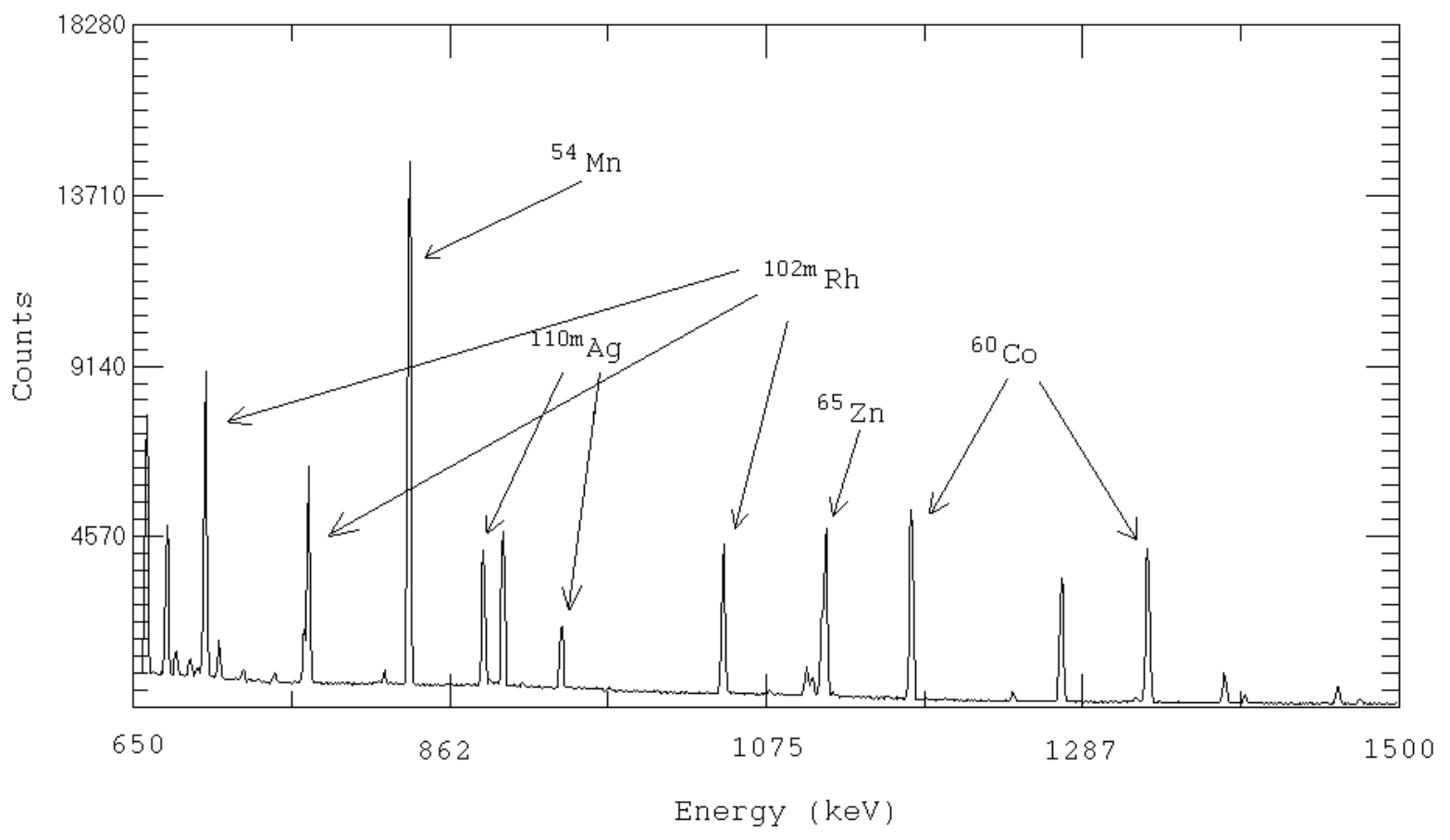

FIGURE 3(b): $\gamma$-ray spectrum ( $861228 \mathrm{sec}$ live time) recorded for the sample irradiated at $23 \mathrm{GeV} 2.83$ years after irradiation: peaks of interest 